\title{
Whole exome sequencing for handedness in a large and highly consanguineous family
}

\author{
Tulya Kavaklioglu $^{\mathrm{a}}$, Muhammad Ajmal ${ }^{\mathrm{b}}$, Abdul Hameed ${ }^{\mathrm{b}, *}$, Clyde Francks $^{\mathrm{a}, * * *}$ \\ ${ }^{a}$ Language and Genetics Department, Max Planck Institute for Psycholinguistics, Nijmegen, The Netherlands \\ ${ }^{\mathrm{b}}$ Institute of Biomedical and Genetic Engineering (IBGE), 24-Mauve Area, G-9/1, Islamabad, Pakistan
}

\section{A R T I C L E I N F O}

\section{Article history:}

Received 22 July 2015

Received in revised form

5 November 2015

Accepted 10 November 2015

Available online 12 November 2015

Keywords:

Handedness

Asymmetry

Linkage

Exome sequencing

Consanguineous

\begin{abstract}
A B S T R A C T
Pinpointing genes involved in non-right-handedness has the potential to clarify developmental contributions to human brain lateralization. Major-gene models have been considered for human handedness which allow for phenocopy and reduced penetrance, i.e. an imperfect correspondence between genotype and phenotype. However, a recent genome-wide association scan did not detect any common polymorphisms with substantial genetic effects. Previous linkage studies in families have also not yielded significant findings. Genetic heterogeneity and/or polygenicity are therefore indicated, but it remains possible that relatively rare, or even unique, major-genetic effects may be detectable in certain extended families with many non-right-handed members. Here we applied whole exome sequencing to 17 members from a single, large consanguineous family from Pakistan. Multipoint linkage analysis across all autosomes did not yield clear candidate genomic regions for involvement in the trait and single-point analysis of exomic variation did not yield clear candidate mutations/genes. Any genetic contribution to handedness in this unusual family is therefore likely to have a complex etiology, as at the population level.
\end{abstract}

(c) 2015 Elsevier Ltd. All rights reserved.

\section{Introduction}

Hand preference in humans is strongly biased to the right at the population level, and is the most overt human behavioural lateralization, with only approximately 1 in every 10 people being left-handed (Hardyck and Petrinovich, 1977). Hand preference is commonly assessed by item-based questionnaires such as the Edinburgh Handedness Inventory (EHI) (Oldfield, 1971), which produces a spectrum of hand preference in the population that has a bimodal distribution. Roughly $1 \%$ of adults show intermediate levels of hand preference defined as ambidextrous, while the great majority show a preference for one hand over the other when considered across multiple tasks (Ocklenburg et al., 2014a).

Behavioural lateralization is visible at early stages of human development. A majority of foetuses at gestational week 10 have been observed by ultrasound scanning to move their right arms more than their left (Hepper et al., 1998), in a proportion strikingly similar to adult rates of handedness. A longitudinal study also

\footnotetext{
* Corresponding author. Institute of Biomedical and Genetic Engineering, 24Mauve Area, G-9/1, Islamabad, Pakistan.

*** Corresponding author: Max Planck Institute for Psycholinguistics, Wundtlaan 1, 6525XD Nijmegen, The Netherlands.

E-mail addresses: ahameed0786@hotmail.com (A. Hameed), Clyde.Francks@mpi.nl (C. Francks).
}

found that foetal 'thumb sucking' at gestational age 15 was predictive of handedness at 10-12 years (Hepper et al., 2005). These behavioural lateralizations in utero occur before brain anatomical lateralization becomes apparent in language-related regions around the Sylvian fissure during the second trimester of gestation, and strongly indicate a genetic-developmental program for human brain and behaviour that is inherently lateralized from embryo onwards (Francks, 2015; Willems et al., 2014).

Indeed handedness is subtly related to functional language lateralization, hinting at overlapping genetic-developmental origins for these traits. While roughly $85 \%$ of humans have left-lateralized language dominance, a higher percentage of left-handers than right-handers have been observed with atypical (reduced or reversed) language lateralization, as assessed by functional transcranial Doppler sonography (fTCD) (Knecht et al., 2000) or functional magnetic resonance imaging (fMRI) (Mazoyer et al., 2014). A recent study, again using fTCD, found that the correlation between left-handedness and atypical functional language lateralization in extended families was $r=0.28$ (Somers et al., 2015). Another recent study, this time based on functional Magnetic Resonance Imaging (fMRI), found that fully reversed (i.e. rightward) functional lateralization for language was found in roughly $7 \%$ of lefthanders, but not at all in right-handers (Mazoyer et al., 2014).

Brain and behavioural asymmetries are widespread across many vertebrate clades, and several species of mammals have 
shown evidence for population-level handedness or pawedness. Subtle population-level paw preference in reaching tests has been observed in inbred mice (Waters and Denenberg, 1994), although the subtlety of these lateralizations required large samples to detect them, and they varied in leftward versus rightward direction depending on the specific task (Waters and Denenberg, 1994). Rats have shown a stronger population-level bias (73\% right paw preference) than mice (Guven et al., 2003). Apes have also shown evidence for population-level handedness, and some structural brain lateralizations similar to those found in regions important for language in humans (Cantalupo et al., 2009; Hopkins, 2013; Hopkins et al., 2011; Lyn et al., 2011; Meguerditchian et al., 2013). Target animacy may play a role in these preferences (Forrester et al., 2011, 2012). These various mammalian species may prove to be useful models for understanding aspects of the genetics and development of human brain lateralization, although they have been barely, or not at all, studied in this regard (Francks, 2015). The right hand is also argued to have been dominant in Neanderthals, through interpreting patterns of scratches on the incisor and canine teeth of fossils (Frayer et al., 2012).

A meta-analysis study of thousands of Australian and Dutch twin families found the heritability of handedness to be close to 25\% (Medland et al., 2009a), which was measured with high-accuracy and strong statistical significance due to the large sample size involved. Single-gene models for handedness have been proposed and extensively discussed in the literature, including the 'right-shift' hypothesis of Annett (2003) (Annett and Alexander, 1996), the 'dextral/chance' hypothesis of McManus (1985), and a model proposed by Klar (1996) which assumes a recessive mode of genetic inheritance. These models integrate the concept of fluctuating asymmetry, in which loss-of-function genotypes at a hypothetical genomic locus result in randomization of lateralized brain development on the left-right axis affecting handedness. It is notable that mutations of certain genes involved in ciliary function are known to cause randomization in the direction of visceral lateralization (Hamada et al., 2002; Levin, 2005), with half of patients manifesting situs inversus of visceral organs and half the normal pattern.

However, a genome-wide association study (GWAS) based on 3940 twins resulted in no individual locus significantly associated to handedness after correction for multiple testing across the genome (Armour et al., 2014). This GWAS study was adequately powered to detect a major-genetic effect on handedness, if it was due to common variation in the genome. Therefore the authors estimated that at least 40 different genetic variants would be required to explain the heritable component of handedness in the population, in other words that locus and/or allelic heterogeneity was likely to be involved (Armour et al., 2014; McManus et al. 2013). A preliminary report of a GWAS from the ENGAGE Handedness Consortium, based on 23,443 subjects, also did not indicate significant evidence for association (Medland et al., 2009b).

A recently conducted linkage analyses on 37 Dutch families also did not identify genomic regions linked significantly to handedness, which was performed under the model of inheritance proposed by McManus, i.e. involving an additive genetic contribution and fluctuating asymmetry (Somers et al., 2015). This study used heterogeneity linkage analysis, which allows for possible differences in genetic effects in the separate families. An earlier linkage study based on 25 nuclear families, performed under Klar's recessive model, also found no significant results (Van Agtmael et al., 2003). A linkage study based on relative hand skill (i.e. lateralized motor performance) found suggestive loci of interest, but again no evidence for there being only one major-genetic effect on handedness in the population (Francks et al., 2002). A genome-wide linkage analysis of extended Mexican-American families also did not identify genomic regions significantly linked to handedness, and the suggestively linked regions were noted to be devoid of obvious candidate genes (Warren et al., 2006).

The combined, very small effects of many thousands of polymorphisms may also affect handedness, together with unknown environmental factors (one known factor is the phenomenon, more prevalent historically, of encouraging or forcing left-handers as children to do things with their right hands (Grabowska et al., 2012; Kloppel et al., 2010)). Initial findings of small individual genetic effects involve the genes Leucine-Rich Repeat Transmembrane neuronal 1 (LRRTM1) and Proprotein Convertase Subtilisin/ Kexin Type 6 (PCSK6), which were both found in a set of families in which at least one sibling had dyslexia, and using measures of lateralized manual performance based on peg moving (Arning et al., 2013; Francks et al., 2007; Scerri et al., 2011). LRRTM1 is a transmembrane protein involved in the differentiation of excitatory synapses (Linhoff et al., 2009). It is not known if LRRTM1 contributes to the development of brain lateralization. PCSK6 is a protease that cleaves NODAL, a member of the transforming growth factor beta (TGF $\beta$ ) superfamily involved in visceral organ lateralization, but again with no functional evidence for a role in brain lateralization. The PCSK6 gene has also been proposed to affect the degree rather than the direction of lateralized hand performance (Arning et al., 2013). In general it is not clear that visceral and brain lateralization are closely linked developmentally, because people with situs inversus (visceral organs reversed on the left-right axis), and having the genetic condition Primary Ciliary Dyskinesia (PCD), did not show changes in rates of left-handedness or left-lateralized auditory language dominance in the largest studies of these issues to have been performed (McManus et al., 2004; Tanaka et al., 1999). Another genetic study of handedness focused on a repeat-length polymorphism at the androgen receptor locus on chromosome $\mathrm{X}$, and found that the number of repeats was associated with handedness (Arning et al., 2015). However, each of these genetic findings requires further validation. Additional small genetic effects, potentially influencing aspects of brain and behavioural lateralization other than handedness, have been reviewed elsewhere (Francks, 2015; Ocklenburg et al., 2014b).

Although the above genetic linkage and association studies of handedness appear to rule out major genetic effects that are due to common variation in the genome, it remains possible that certain individual, extended families with elevated rates of non-righthandedness may have relatively rare or unique genetic sub-forms of the trait that are affected disproportionately by a single gene mutation. Although one previous study used heterogeneity linkage analysis (Somers et al., 2015), few of the individual families in that study were large enough to provide adequate statistical power to detect significant linkage, in the case of extensive heterogeneity of effects between families. In the present study we have considered a recessive major-genetic model in a single, large, extended family from Pakistan with multiple instances of consanguineous marriage and an elevated rate of non-right-handedness in the younger generations. Consanguinity is known to increase the chances of having recessive genetic traits and disorders in offspring, since offspring are likely to inherit two copies of sections of the genome identical-by-descent, and homozygous for any genetic mutations within those regions.

We performed whole exome sequencing using next generation DNA sequencing in 17 members from this family, which allowed us to perform not only a classical recessive linkage scan, but also systematic screening of DNA sequence variants affecting the protein-coding portion of genes in potentially linked regions of the genome. The exome is the roughly $1-2 \%$ of the genome that codes for proteins. In our analysis we allowed for incomplete penetrance (90\%; i.e. allowing a $10 \%$ chance that a recessive mutation might not cause non-right-handedness) and 10\% phenocopy (i.e. 
allowing people without the recessive mutation to still develop non-right-handedness at roughly the rate in the general population). We additionally tested a recessive model similar to that proposed by Klar (1996), by modelling 50\% penetrance (again with $10 \%$ phenocopy), i.e. that the recessive mutation would result half the time in the normal pattern of right-handedness, and half the time in non-right-handedness (akin to recessive mutations causing situs inversus with $50 \%$ probability in primary ciliary dyskinesia). We also tested for linkage under an additive model akin to the 'dextral/chance' model of McManus (1985). Finally, we performed a set of exploratory analyses that were intended to ensure that our main analyses had not missed clear signals of linkage in the family. These allowed for a reduced population prevalence of left-handedness in Pakistan, or a higher-penetrance additive effect.

\section{Materials and methods}

\subsection{Subjects and handedness assessment}

Family 3LHAND is a family from Pakistan, with 6 generations and 89 members as shown in Fig. 1. Handedness of 35 of the family members was assessed with the 10 item version of EHI (Oldfield, 1971), and 14 of these members (40\%) were either in the ambidextrous or left-handed range, with a clear separation between righthanded and non-right-handed members (Fig. 2). Based on this distribution we classified members with negative EHI scores as non-right-handed, and those with EHI scores above 50 as right-handed. No subjects had EHI scores between 0 and +50 . We did not attempt to further sub-divide the non-right-handers into lefthanders and ambidextrous people. This was for two reasons: first, there was no clear distinction in the distribution of EHI scores in this family to make this division (Fig. 2), and second, left-handers are generally under more cultural pressure than right handers to carry out certain tasks with their non-preferred hand, which could potentially cause skewing of the left-handed EHI distribution towards the ambidextrous range. The rate of $40 \%$ non-right-handed is high compared to the general human population (Hardyck and Petrinovich, 1977; Perelle and Ehrman, 1994). We were not aware of reliable and recent data on the prevalence of non-right-handedness in the Pakistan population specifically, but cultural suppression may make the prevalence even lower than in Western countries (Masud and Ajmal, 2012). The handedness of the older generations and deceased people in the family was not assessed. For our analysis, any individuals who had not completed an EHI themselves were considered as having unknown phenotypes. The overall family tree has 10 consanguineous marriages (Fig. 1). No family members were reported to have any other remarkable behavioural traits, nor had they been diagnosed with schizophrenia, dyslexia, low-IQ or neurological diseases such as early onset Alzheimer's or Parkinson's disease. There was no family history of any inherited disease/ disorder. All 35 participating members were healthy with normal school/work lives. DNA samples were extracted from blood (phenol extraction). Seventeen of

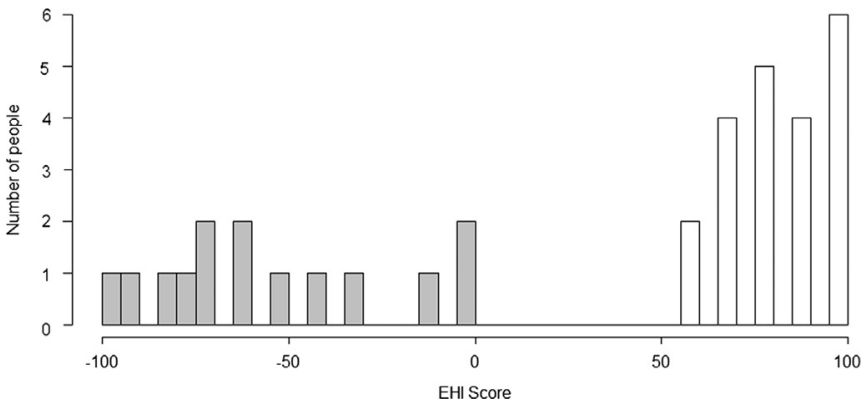

Fig. 2. Frequency distribution of hand preference scores based on the EHI in 35 family members. Hand preference scores classified as belonging to non-righthanders are indicated by grey shading. The EHI score can range from fully lefthanded $(-100)$ to fully right-handed $(+100)$.

these members were selected for exome sequencing (Fig. 1). Amongst these 17 members, 8 were non-right-handed and 9 were right-handed. The study was approved by the Institute of Biomedical and Genetic Engineering Ethical Committee Islamabad.

\subsection{Next generation sequencing}

DNA samples collected from 17 members of the 3LHAND family were used for whole exome sequencing by the genomics research organization and service company 'BGI' (Hong Kong/Shenzhen) using Illumina's HiSeq 2000 technology (BGI, 2015; Illumina, 2015). Exome capture was done with the Agilent SureSelect All Human Exon V4 (51 megabase) array (SureSelect Performance, 2015). Sequencing was at 100 times average coverage depth with library insert size 150-200 base pairs. Depending on the amount of available DNA, one or two libraries were constructed for each sample.

\subsection{NGS data processing}

\subsubsection{Alignment and pre-processing}

Clean raw reads $(\sim 94 \%$ of the total reads - i.e. excluding adaptor sequence low-quality bases for more than $50 \%$ of the bases in a read, and unknown bases for more than $10 \%$ of bases in a read) were mapped onto the human reference genome (UCSC Genome Browser hg19) using the software BWA (Li and Durbin, 2009). The mean sequence read length was $\sim 88$ base pairs. $99.3 \%$ of the target exome was covered by at least a 10 times sequence read depth. PCR duplicated reads were marked using Picard (Picard, 2015).

\subsubsection{Variant calling and annotation}

Raw reads were reordered by use of the software Picard (Picard, 2015), and realignment around indels (insertion/deletions) and base quality control recalibration

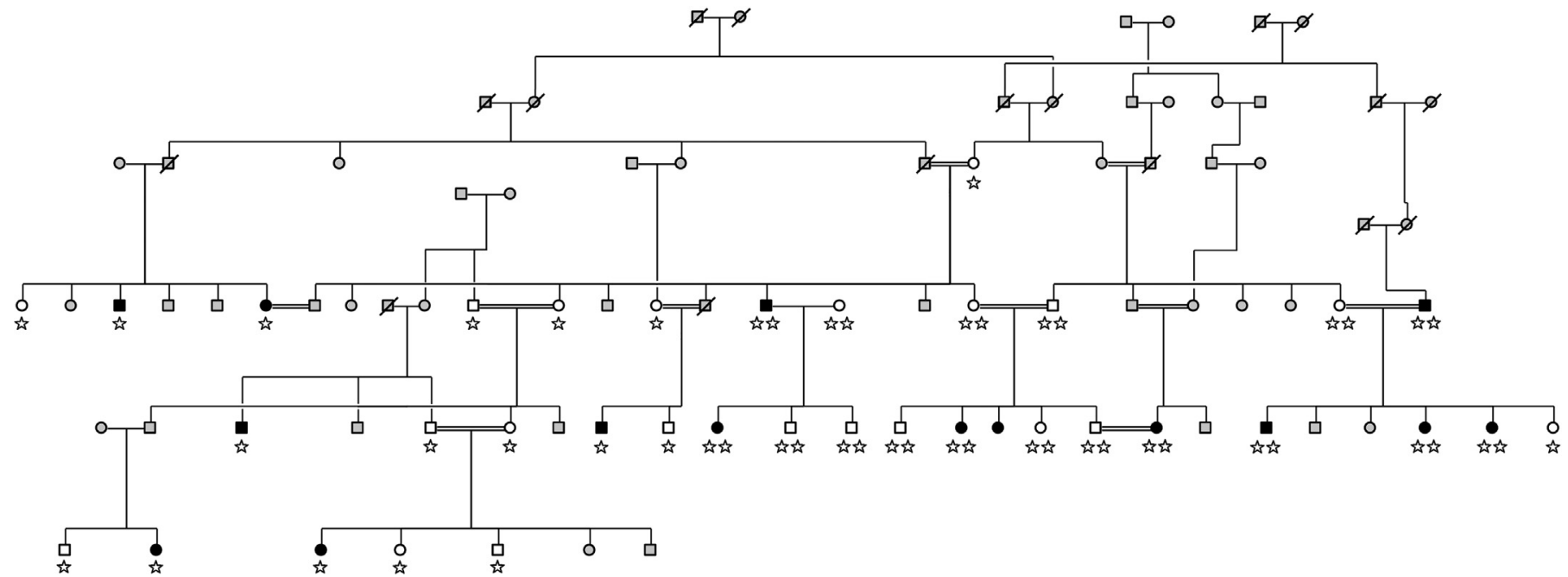

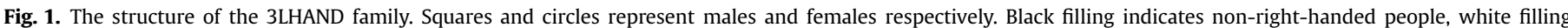

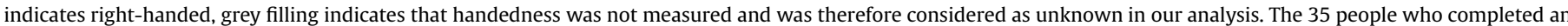

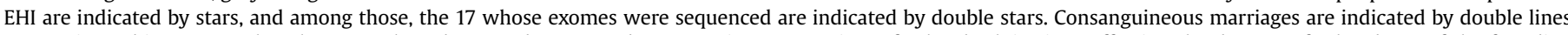

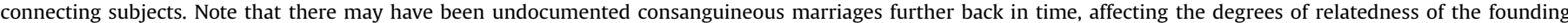
individuals. 
was performed using the software package GATK (DePristo et al., 2011; McKenna et al., 2010; Van der Auwera et al., 2013). The software SAMtools (Li et al., 2009) was used for reading binary (bam) files. A target interval file was generated by using the UCSC Genome Browser (UCSC Genome Browser on Human, February 2009 (GRCh37/hg19) Assembly, 2015) (the reference genome was hg19, and 100 base pairs surrounding the exons were also included). Genetic variants in the sequence data were called using the HaplotypeCaller tool of GATK, using its default settings and pipeline, except that we set the confidence thresholds as: stand_call_conf $=50.0$, stand_emit_conf $=10.0$. More accurate variant calls can be achieved by including data from larger numbers of subjects simultaneously, and we therefore ran this process using the 17 sequenced members of family 3LHAND together with 10 additional subjects from another project that had been sequenced with the identical protocol and in the same batch. A total of 266,171 variants across all subjects were called in this way. 40,106 out of these 266,171 variants were not present in the dbSNP database. The number of variants called per sample had a mean of $232,704(\min =214,654$, $\max =255,524)$. Quality filtering procedures were completed by using VCFtools (Danecek et al., 2011): Variants with less than $10 \times$ read depth and a quality score of 50 were filtered out, and variants not assigned to specific chromosomes were removed. The 264,714 remaining variants were annotated with wANNOVAR (the web based version of ANNOVAR) (Wang et al., 2010).

\subsection{Multipoint parametric linkage}

Multipoint linkage analysis requires a set of polymorphisms spaced roughly evenly over the genome but which are not in linkage disequilibrium (LD) with one another. This analysis does not require that the causative variant itself is retained in the multipoint linkage map. For this purpose we first removed indels and then pruned the variants for pairwise LD (using the software PLINK (Purcell et al., 2007) with default parameters, window size $=50$, SNP shift $=5$, variance inflation factor $=2$ ). A total number of 50,045 exomic variants with rs identity numbers in the dbSNP database (Sherry et al., 2001) were subjected to LD pruning. LD was defined according to a set of 1303 Dutch subjects from the Brain Imaging Genetics (BIG) dataset (Franke et al., 2010), so that only the variants genotyped or imputed in BIG could be selected for the pruning. Variants with anything other than 2 alleles were also removed. This resulted in 14,103 independent (un-correlated) variants. A genetic map was then created using a tool within the SNP/Max system of BC Platforms (2015). Variants showing Mendelian errors were removed using PedCheck (O'Connell and Weeks, 1998). A further pruning step based on polymorphism information content (PIC) within the family (minimum distance between markers $=0.2 \mathrm{cM}$, most informative marker selected within $0.1 \mathrm{cM}$ ) resulted in 4549 variants. Note that this set of variants merely provided a scaffold map of highfrequency polymorphisms for performing unbiased multipoint linkage analysis, after which we would interrogate every bi-allelic, exonic, non-synonymous variant within putatively linked regions, without any of this filtering (see Section 2.5 below).

Multipoint parametric recessive autosomal linkage analysis was carried out using the program SwiftLink (Medlar et al., 2013). This program handles multiple consanguineous marriages without 'loop breaking', and therefore fully exploits the structure of such families for recessive linkage analysis. For all linkage analysis in this study, we used the full family structure of 89 individuals, while only assigning phenotypes for the 35 who had completed the $\mathrm{EHI}$, and only assigning genotypes for the 17 phenotyped subjects who had also undergone exome sequencing. For our primary analysis, LOD scores ( $\log 10$ of the odds of linkage) were calculated using an assumed $90 \%$ penetrance for non-right-handedness for a recessive causative mutation. A phenocopy rate of $10 \%$ was specified to reflect the approximate population frequency of non-right-handedness. We were able to manually simulate a recessive mutation co-segregating near perfectly with non-right-handedness in this family. The simulated variant had a frequency of 0.2 among the family's founders, i.e. it needed to be present in 8 out of the 19 unrelated founder individuals in heterozygous form. We therefore specified an assumed mutation allele frequency of 0.2 in our linkage analysis. Note that this does not necessarily assume the mutation is present at 0.2 frequency in the general Pakistani population. It could be much rarer in the population, but be at an elevated frequency in the founders of this family because of undocumented consanguinity higher up in the family tree that affected the relatedness of founders, and/or because some founders may have come from a particular regional sub-population. Both scenarios are likely for this family.

We also performed a multipoint linkage screen under a fully recessive mode with $50 \%$ penetrance for the mutant homozygous genotype, and a multipoint linkage screen under an additive model for which the homozygous mutation had $50 \%$ penetrance and the heterozygote had 25\% penetrance (phenocopy rate was again set to $10 \%$; see Section 1 for the rationale). Mutation frequency was again set to 0.2 . We considered these as secondary analyses for two reasons. First, in order for a relatively weakly penetrant allele to explain the pattern of left-handedness in this particular family, it would need to be the major (i.e. more frequent) allele among the founders, which is unlikely even if there was undocumented consanguinity higher up in the family tree, and given the overall population frequency of non-right-handedness together with the fact that genome-wide association scanning has not identified major-genetic effects on handedness arising from common genetic variation (see Section 1). Second, our phenotype of non-right- handedness as a single group in this particular family, i.e. arising because ambidextrality was continuous with left-handedness in the EHI distribution for this family (Fig. 2), does not necessarily support models based on fluctuating asymmetry in the same way as a clear left-versus-right distinction might.

\subsection{Single-point parametric linkage}

Single-point linkage analysis was conducted for all bi-allelic, non-synonymous (i.e. protein altering) exonic variants that were located within genomic regions showing LOD scores $>1$ in the multipoint analysis, whether or not they had been included in the multipoint linkage map, and without regard to their presence, absence or reported frequencies in on-line databases. Thus the full spectrum of rare, private, ancestry-specific and common variants were tested from within regions of multipoint linkage showing LOD scores $>1$. As SwiftLink only performs multipoint analysis and cannot test individual variants for linkage, the program Pseudomarker was used for single-point analysis (Gertz et al., 2014; Goring and Terwilliger, 2000; Hiekkalinna et al., 2011). This program deals with consanguineous marriages by 'loop breaking'. We used the same linkage model parameters for single-point analysis as for the multipoint analysis that had implicated a given genomic region (penetrance, phenocopy, mutation frequency).

\subsection{Maximum LOD scores possible}

We used our manually simulated variant, which showed complete homozygous co-segregation with non-right-handedness among the 17 subjects that we sequenced (8 non-right-handed, 9 right-handed), in order to assess the maximum LOD scores that we could have achieved with this family by having sequenced these 17 members. The manually simulated variant was entered into linkage analysis the same way as real variants, except that for multipoint analysis we simulated ten such variants in a row along a dummy chromosome, each at $1 \mathrm{cM}$ genetic distance from the previous one. For multipoint analysis under the $90 \%$ penetrance model, the maximum LOD score was 2.17 , under the fully recessive $50 \%$ penetrance model it was 1.16 , and under the additive model described above it was 0.52 . For single-point analysis under the $90 \%$ penetrance model, the maximum LOD score was 1.43 , under the recessive $50 \%$ penetrance model it was 0.69 , and under the additive model it was 0.33 . Note that a dummy variant perfectly co-segregating with non-right-handedness in homozygous form is a priori not well fitted for the models with lower penetrance, because these models require that a large number of right-handers would also be homozygous for the mutation. However, under such models, as noted above, the frequency of the mutation would need to be unrealistically high in the founders of this family (it would need to be the more frequent allele in the founders).

\subsection{Exploratory analyses under different models}

As our analyses did not yield significant evidence for linkage or implicate specific variants in non-right-handedness (see below), we performed additional, exploratory multipoint analyses under various different model parameters to rule out the possibility of having missed relevant signals in the data. First, due to possible cultural suppression of left-handedness in Pakistan (Masud and Ajmal, 2012), we repeated the three linkage analyses described above (Section 2.4), except that we reduced the phenocopy rate from $10 \%$ to $3 \%$. Second, we applied a higher-penetrance additive model for which the homozygous mutation had $80 \%$ penetrance, the heterozygote had $40 \%$ penetrance, and with a $10 \%$ phenocopy rate for the homozygote wild-type. For all of these exploratory analyses, a mutation frequency of 0.2 was used. After multipoint linkage analysis, single point LOD scores were calculated for all bi-allelic, exonic, and non-synonymous variants located within the linkage regions that showed multipoint $\operatorname{LOD}>1$, and by applying the same parameters used in the respective multipoint linkage analysis that implicated a given region.

\section{Results}

\subsection{Multipoint linkage across the genome}

Under the model of $90 \%$ penetrance for a homozygous mutation, no regions of the genome showed multipoint linkage with non-right-handedness at LOD scores greater than 1.35 (Fig. 3). This was considerably lower than the maximum possible LOD score that this analysis could have yielded $(\mathrm{LOD}=2.17)$, as assessed by our simulation of a perfectly co-segregating homozygous mutation with non-right-handedness among the 17 sequenced subjects. Only four regions of the genome showed multipoint LOD scores above 1 , which were on chromosomes 2, 7, 9, and 14 (Fig. 3). 


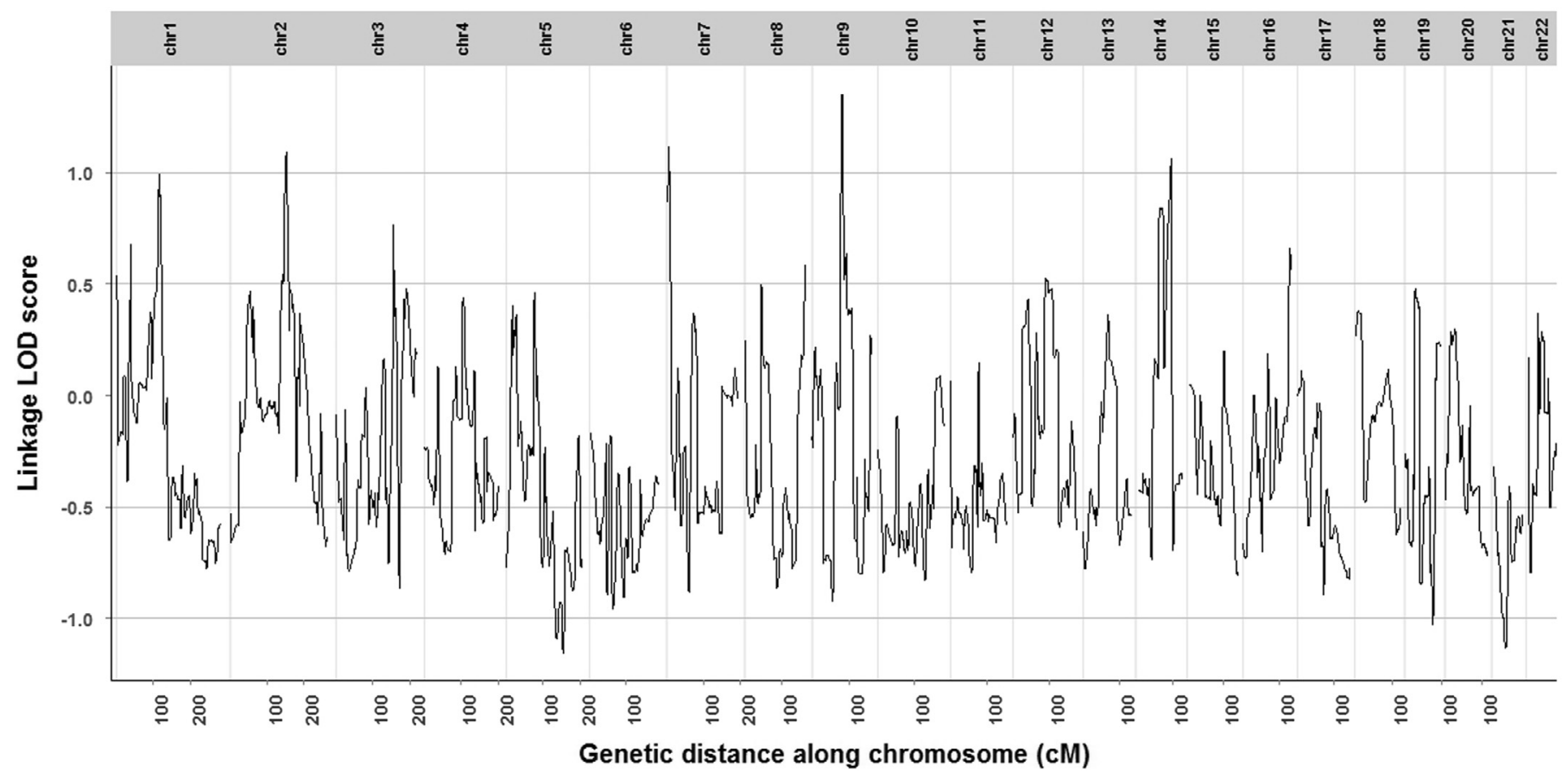

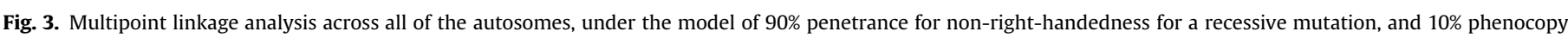
rate. The $x$-axis shows genetic distances (in Haldane centimorgans) within each chromosome; the $y$-axis shows the linkage LOD scores.

Under the model of $50 \%$ penetrance for a fully recessive homozygous mutation, no regions of the genome showed multipoint linkage with non-right-handedness at LOD scores greater than 0.88 (results not shown). Under the additive model described above, no region of the genome showed a LOD score greater than 0.53 (results not shown).

\subsection{Single-point linkage of protein-altering variants}

Within the four genomic regions showing multipoint linkage LOD scores greater than 1 under the model with $90 \%$ penetrance, there were 58 bi-allelic variants in 24 different genes that were exonic and non-synonymous (i.e. DNA sequence variants that cause changes in protein amino acid sequences). Each of these variants was tested for single-point linkage with handedness, under the same model parameters that implicated the genomic regions in multipoint analysis. Only three of these variants yielded single-point linkage $p$ values below the nominal significance level (i.e. not adjusted for multiple testing) of $p=0.05$ (Table 1 ). None of the three linkages were significant in the context of multiple testing. All three variants were already recorded in existing databases of polymorphisms (i.e. none were novel/unique to this family) (1000 Genomes Project Consortium et al., 2012; NHLBI Exome Sequencing Project (ESP) Exome Variant Server, 2015). Amongst these 3 variants, only one (rs1061566) yielded a singlepoint LOD score above 1 and might therefore in principle have

Table 1

The three exonic non-synonymous variants that showed nominally significant single-point linkage to non-right-handedness ( $p$ values $<=0.05$ ), and were present within regions of multipoint linkage with LOD scores greater than 1 , under the model of $90 \%$ penetrance for a homozygous mutation, and a phenocopy rate of $10 \%$.

\begin{tabular}{llllll}
\hline Chromosome & Position & Variant name & Gene & $\begin{array}{l}\text { Single- } \\
\text { point LOD }\end{array}$ & p Value \\
\hline 2 & $137,814,138$ & rs60939389 & THSD7B & 0.659476 & 0.040696 \\
7 & $2,649,704$ & rs3735109 & IQCE & 0.645796 & 0.042308 \\
7 & $2,649,777$ & rs1061566 & IQCE & 1.157612 & 0.010486 \\
\hline
\end{tabular}

explained the multipoint linkage spanning this genomic region, as a recessive mutation for non-right-handedness. This variant is located within the IQCE gene on chromosome $7 \mathrm{p} 22.3$, and is responsible for a threonine to methionine amino acid change in the protein at location 625. However, for this variant, non-righthandedness was linked to the major (i.e. more common) allele as reported in the 1000 Genomes database $(1000$ Genomes Project Consortium et al., 2012) for the Pakistani population (86\% major allele frequency). It is therefore unlikely that this polymorphism can be highly penetrant for non-right-handedness, otherwise nonright-handedness would exist at a higher frequency in the population. For this reason we regard this non-significant finding as statistical noise.

\subsection{Exploratory analyses under other models}

When modelling a low-phenocopy rate of 3\% to reflect possible cultural suppression of left-handedness in Pakistan, the highest multipoint LOD score in the genome was 1.29 for the model with $50 \%$ penetrance for a recessive mutation. When modelling the higher-penetrance additive model (Section 2.7) the highest LOD score in the genome was 0.78 . Single point linkage analysis of all bi-allelic, exonic, and non-synonymous variants located within the regions that showed multipoint linkage LOD $>1$ in these exploratory analyses, revealed no individual variants with single point LOD scores greater than 1.

\section{Discussion}

The molecular genetic basis of human brain lateralization is unknown. Identifying genetic variants linked to human handedness has the potential to increase our understanding of the ontogenesis of lateralized functions in the brain, which prominently include motor control and language, but also a range of other processes such as visuospatial cognition (Gotts et al., 2013; Herve et al., 2013; Renteria, 2012; Tomasi and Volkow, 2012). Alterations of brain lateralization and handedness have also been reported to 
associate with cognitive disorders including dyslexia and Specific Language Impairment (SLI), and psychiatric disorders including schizophrenia and autism, although not in all clinical populations (Deep-Soboslay et al., 2010; Preslar et al., 2014; Renteria, 2012). Genes involved in brain lateralization may be relevant for some forms of these conditions.

In this study we used whole exome sequencing of seventeen members of a large, extended family to identify genomic regions and individual genetic variants linked to non-right-handedness. Our primary hypothesis for linkage was under a strongly penetrant recessive model of inheritance, which was appropriate to the highly consanguineous nature of the family, the relatively highfrequency of non-right-handedness within the family, and the distribution of hand preference scores among non-right-handed members of the family. The use of next-generation sequencing, and the analysis of a single, large, consanguineous family, were novel aspects of our study, neither of which have previously been applied in genetic investigations of handedness. Our approach was suited to the possibility that unusual families may have relatively rare, or even unique, major-genetic subforms of non-right-handedness. Our focus on subjects from Pakistan was also novel, as previous genetic studies of handedness (see Section 1) have made use of predominantly or wholly European-descent populations or families, and may therefore have missed genetic effects that are present in other populations but weak or absent in Europeandescent populations.

By simulating a dummy marker with perfect co-segregation of a homozygous allele with non-right-handedness among the seventeen subjects who we sequenced from the family, we were able to obtain a maximum possible LOD score of 2.17 in the family, under a model assuming 90\% penetrance for the homozygous mutations, $10 \%$ phenocopy, and $20 \%$ mutation allele frequency among the family's founders. Had such a mutation been present in our real data, we would most likely have detected it through our strategy of multipoint linkage analysis across the genome followed by single-point analysis of all protein-altering variants within putatively linked genomic regions. As it was, our multipoint analysis yielded a maximum linkage that was considerably lower than that which was attainable in this family, indicating that no such mutation was present in the family. Within genomic regions showing multipoint LOD scores greater than 1, we found no protein-altering variants that showed convincing linkage to nonright-handedness and which could realistically act as penetrant mutations in the Pakistani or world populations. We also considered two models of fluctuating asymmetry but these analyses yielded even weaker linkage results in this family. Additional exploratory analyses that allowed for reduced population prevalence, or a higher-penetrance additive effect, also did not result in any variant of potential interest. Note that a LOD score of 3 is conventionally used as a threshold to declare significant linkage in a genome-wide scan of this kind. Although our primary model only permitted a maximum LOD score of 2.17 , if we had found such a linkage, together with a likely causative variant within the corresponding genomic region (such as an overtly disruptive, rare mutation in a neurally expressed or key developmental gene), then the combination of evidence would have motived further research in population datasets and other families, as well as gene functional analysis. On the basis of our data we conclude that nonright-handedness in this family is likely to be an etiologically complex trait, potentially involving locus and allelic heterogeneity, polygenicity, and unknown environmental influences, as has been indicated for non-right-handedness at the population level (see Section 1).

While protein-altering DNA variants are a priori the most likely class of variants to have highly penetrant effects on human traits, it is possible that our focus on exomic, protein-altering variation may have missed other classes of potentially relevant sequence variation. Non-exomic variation can be present in genomic regulatory elements, which can affect the amounts and developmental timing of, for example, gene expression and protein translation (Battle et al., 2015; Brucato et al., 2015). Another possibility is that a structural variant, for example a larger scale deletion or duplication, was present in the genomes of some of the family members, and not detected by the exome sequencing. A further possibility is that our exome capture and sequencing missed a putatively causal exomic variant for the trait, as the protocol that we used is known to miss between $5-10 \%$ of proteincoding DNA variation (Gnirke et al., 2009; SureSelect Performance, 2015). However, even if there was a non-exonic, missed exonic, or structural mutation in the family, which had a high-recessive penetrance for non-right-handedness, it would still have most likely resulted in a detectable linkage signal in multipoint analysis, which uses only a scaffold map of polymorphisms to assess broad genomic regions and does not require the causative variant to be included in the map.

It remains possible that other large, extended families with elevated rates of left-handedness may be affected by rare or unique but relatively penetrant mutations, acting in various ways with regard to allelic dominance, and with various degrees of penetrance. We continue to advocate the collection of such families for combined linkage and exome or whole genome sequencing analysis, which holds the potential to identify key genes involved in human brain lateralization.

\section{Acknowledgements}

We thank Simon E. Fisher for supporting the project and providing feedback on the draft manuscript. This work was funded by the Max Planck Society (Germany). We are also thankful to the family members for voluntarily participating in this study.

\section{References}

1000 Genomes Project Consortium, Abecasis, G.R., Auton, A., Brooks, L.D., DePristo, M.A., Durbin, R.M., et al., 2012. An integrated map of genetic variation from 1092 human genomes. Nature 491 (7422), 56-65. http://dx.doi.org/10.1038/ nature 11632.

Annett, M., 2003. Cerebral asymmetry in twins: predictions of the right shift theory. Neuropsychologia 41 (4), 469-479.

Annett, M., Alexander, M.P., 1996. Atypical cerebral dominance: predictions and tests of the right shift theory. Neuropsychologia 34 (12), 1215-1227.

Armour, J.A., Davison, A., McManus, I.C., 2014. Genome-wide association study of handedness excludes simple genetic models. Heredity 112 (3), 221-225. http: //dx.doi.org/10.1038/hdy.2013.93.

Arning, L., Ocklenburg, S., Schulz, S., Ness, V., Gerding, W.M., Hengstler, J.G., et al., 2013. VNTR polymorphism is associated with degree of handedness but not direction of handedness. PLoS One 8 (6), e67251. http://dx.doi.org/10.1371/ journal.pone.0067251.

Arning, L., Ocklenburg, S., Schulz, S., Ness, V., Gerding, W.M., Hengstler, J.G., et al., 2015. Handedness and the X chromosome: the role of androgen receptor CAGrepeat length. Sci. Rep. 5, 8325. http://dx.doi.org/10.1038/srep08325.

Battle, A., Khan, Z., Wang, S.H., Mitrano, A., Ford, M.J., Pritchard, J.K., Gilad, Y., 2015. Genomic variation. Impact of regulatory variation from RNA to protein. Science 347 (6222), 664-667. http://dx.doi.org/10.1126/science.1260793.

BC Platforms, 2015. Retrieved 13.07.15, from $\langle$ http://bcplatforms.com/ $\rangle$.

BGI, 2015. Retrieved 13.07.15, from 〈http://www.genomics.cn/index $\rangle$.

Brucato, N., Guadalupe, T., Franke, B., Fisher, S.E., Francks, C., 2015. A schizophreniaassociated HLA locus affects thalamus volume and asymmetry. Brain Behav. Immun. 46, 311-318. http://dx.doi.org/10.1016/j.bbi.2015.02.021.

Cantalupo, C., Oliver, J., Smith, J., Nir, T., Taglialatela, JP., Hopkins, W.D., 2009. The chimpanzee brain shows human-like perisylvian asymmetries in white matter. Eur. J. Neurosci. 30 (3), 431-438. http://dx.doi.org/10.1111/ j.1460-9568.2009.06830.x.

Danecek, P., Auton, A., Abecasis, G., Albers, C.A., Banks, E., DePristo, M.A., et al., 2011. The variant call format and VCFtools. Bioinformatics 27 (15), 2156-2158. http: //dx.doi.org/10.1093/bioinformatics/btr330. 
Deep-Soboslay, A., Hyde, T.M., Callicott, J.P., Lener, M.S., Verchinski, B.A., Apud, J.A., et al., 2010. Handedness, heritability, neurocognition and brain asymmetry in schizophrenia. Brain 133 (10), 3113-3122. http://dx.doi.org/10.1093/brain/ awq160.

DePristo, M.A., Banks, E., Poplin, R., Garimella, K.V., Maguire, J.R., Hartl, C., et al., 2011. A framework for variation discovery and genotyping using next-generation DNA sequencing data. Nat. Genet. 43 (5), 491-498. http://dx.doi.org/ $10.1038 /$ ng.806.

Forrester, G.S., Leavens, D.A., Quaresmini, C., Vallortigara, G., 2011. Target animacy influences gorilla handedness. Anim. Cogn. 14 (6), 903-907. http://dx.doi.org/ 10.1007/s10071-011-0413-6.

Forrester, G.S., Quaresmini, C., Leavens, D.A., Spiezio, C., Vallortigara, G., 2012. Target animacy influences chimpanzee handedness. Anim. Cogn. 15 (6) 1121-1127. http://dx.doi.org/10.1007/s10071-012-0536-4.

Francks, C., 2015. Exploring human brain lateralization with molecular genetics and genomics. Ann. N. Y. Acad. Sci. http://dx.doi.org/10.1111/nyas.12770/abstract.

Francks, C., Fisher, S.E., MacPhie, I.L., Richardson, A.J., Marlow, A.J., Stein, J.F., Monaco, A.P., 2002. A genomewide linkage screen for relative hand skill in sibling pairs. Am. J. Hum. Genet. 70 (3), 800-805. http://dx.doi.org/10.1086/ 339249.

Francks, C., Maegawa, S., Lauren, J., Abrahams, B.S., Velayos-Baeza, A., Medland, S.E., et al., 2007. LRRTM1 on chromosome 2p12 is a maternally suppressed gene that is associated paternally with handedness and schizophrenia. Mol. Psychiatry 12 (12), 1129-1139. http://dx.doi.org/10.1038/sj.mp.4002053 1057.

Franke, B., Vasquez, A.A., Veltman, J.A., Brunner, H.G., Rijpkema, M., Fernandez, G., 2010. Genetic variation in CACNA1C, a gene associated with bipolar disorder, influences brainstem rather than gray matter volume in healthy individuals. Biol. Psychiatry 68 (6), 586-588. http://dx.doi.org/10.1016/j. biopsych.2010.05.037.

Frayer, D.W., Lozano, M., Bermudez de Castro, J.M., Carbonell, E., Arsuaga, J.L., Radovcic, J., et al., 2012. More than 500,000 years of right-handedness in Europe. Laterality 17 (1), 51-69. http://dx.doi.org/10.1080/1357650X.2010.529451.

Gertz, E.M., Hiekkalinna, T., Digabel, S.L., Audet, C., Terwilliger, J.D., Schaffer, A.A 2014. PSEUDOMARKER 2.0: efficient computation of likelihoods using NOMAD. BMC Bioinform. 15, 47. http://dx.doi.org/10.1186/1471-2105-15-47.

Gnirke, A., Melnikov, A., Maguire, J., Rogov, P., LeProust, E.M., Brockman, W., et al., 2009. Solution hybrid selection with ultra-long oligonucleotides for massively parallel targeted sequencing. Nat. Biotechnol. 27 (2), 182-189. http://dx.doi.org/ 10.1038/nbt.1523.

Goring, H.H., Terwilliger, J.D., 2000. Linkage analysis in the presence of errors IV: joint pseudomarker analysis of linkage and/or linkage disequilibrium on a mixture of pedigrees and singletons when the mode of inheritance cannot be accurately specified. Am. J. Hum. Genet. 66 (4), 1310-1327. http://dx.doi.org/ $10.1086 / 302845$

Gotts, S.J., Jo, H.J., Wallace, G.L., Saad, Z.S., Cox, R.W., Martin, A., 2013. Two distinct forms of functional lateralization in the human brain. Proc. Natl. Acad. Sci. S. A 110 (36), E3435-E3444. http://dx.doi.org/10.1073/pnas.1302581110.

Grabowska, A., Gut, M., Binder, M., Forsberg, L., Rymarczyk, K., Urbanik, A., 2012. Switching handedness: fMRI study of hand motor control in right-handers, lefthanders and converted left-handers. Acta Neurobiol. Exp. (Wars) 72 (4), 439-451.

Guven, M., Elalmis, D.D., Binokay, S., Tan, U., 2003. Population-level right-paw preference in rats assessed by a new computerized food-reaching test. Int. J. Neurosci. 113 (12), 1675-1689. http://dx.doi.org/10.1080/00207450390249258.

Hamada, H., Meno, C., Watanabe, D., Saijoh, Y., 2002. Establishment of vertebrate left-right asymmetry. Nat. Rev. Genet. 3 (2), 103-113. http://dx.doi.org/10.1038/ nrg732.

Hardyck, C., Petrinovich, L.F., 1977. Left-handedness. Psychol. Bull. 84 (3), 385-404.

Hepper, P.G., McCartney, G.R., Shannon, E.A., 1998. Lateralised behaviour in first trimester human foetuses. Neuropsychologia 36 (6), 531-534.

Hepper, P.G., Wells, D.L., Lynch, C., 2005. Prenatal thumb sucking is related to postnatal handedness. Neuropsychologia 43 (3), 313-315. http://dx.doi.org 10.1016/j.neuropsychologia.2004.08.009.

Herve, P.Y., Zago, L., Petit, L., Mazoyer, B., Tzourio-Mazoyer, N., 2013. Revisiting human hemispheric specialization with neuroimaging. Trends Cogn. Sci. 17 (2), 69-80. http://dx.doi.org/10.1016/j.tics.2012.12.004.

Hiekkalinna, T., Schaffer, A.A., Lambert, B., Norrgrann, P., Goring, H.H., Terwilliger, J. D., 2011. PSEUDOMARKER: a powerful program for joint linkage and/or linkage disequilibrium analysis on mixtures of singletons and related individuals. Hum. Hered. 71 (4), 256-266. http://dx.doi.org/10.1159/000329467.

Hopkins, W.D., 2013. Neuroanatomical asymmetries and handedness in chimpanzees (Pan troglodytes): a case for continuity in the evolution of hemispheric specialization. Ann. New. Y. Acad. Sci. 1288, 17-35. http://dx.doi.org/10.1111/ nyas.12109, n/a-n/a.

Hopkins, W.D., Phillips, K.A., Bania, A., Calcutt, S.E., Gardner, M., Russell, J., et al., 2011. Hand preferences for coordinated bimanual actions in 777 great apes: implications for the evolution of handedness in hominins. J. Hum. Evol. 60 (5), 605-611. http://dx.doi.org/10.1016/j.jhevol.2010.12.008.

Illumina, 2015. HiSeq 2000. Retrieved 13.07.15, from 〈http://support.illumina.com/ sequencing/sequencing_instruments/hiseq_2000.html〉.

Klar, A.J., 1996. A single locus, RGHT, specifies preference for hand utilization in humans. Cold Spring Harb. Symp. Quant. Biol. 61, 59-65.

Kloppel, S., Mangin, J.F., Vongerichten, A., Frackowiak, R.S., Siebner, H.R., 2010. Nurture versus nature: long-term impact of forced right-handedness on structure of pericentral cortex and basal ganglia. J. Neurosci. 30 (9), 3271-3275. http://dx.doi.org/10.1523/JNEUROSCI.4394-09.2010.
Knecht, S., Drager, B., Deppe, M., Bobe, L., Lohmann, H., Floel, A., et al., 2000. Handedness and hemispheric language dominance in healthy humans. Brain 123, 2512-2518, Pt 12.

Levin, M., 2005. Left-right asymmetry in embryonic development: a comprehensive review. Mech. Dev. 122 (1), 3-25. http://dx.doi.org/10.1016/j. mod 2004.08.006.

Li, H., Durbin, R., 2009. Fast and accurate short read alignment with burrowswheeler transform. Bioinformatics 25 (14), 1754-1760. http://dx.doi.org/ 10.1093/bioinformatics/btp324.

Li, H., Handsaker, B., Wysoker, A., Fennell, T., Ruan, J., Homer, N., et al., 2009. The sequence alignment/map format and SAMtools. Bioinformatics 25 (16), 2078-2079. http://dx.doi.org/10.1093/bioinformatics/btp352.

Linhoff, M.W., Lauren, J., Cassidy, R.M., Dobie, F.A., Takahashi, H., Nygaard, H.B., et al., 2009. An unbiased expression screen for synaptogenic proteins identifies the LRRTM protein family as synaptic organizers. Neuron 61 (5), 734-749. http: //dx.doi.org/10.1016/j.neuron.2009.01.017.

Lyn, H., Pierre, P., Bennett, A.J., Fears, S., Woods, R., Hopkins, W.D., 2011. Planum temporale grey matter asymmetries in chimpanzees (Pan troglodytes), vervet (Chlorocebus aethiops sabaeus), rhesus (Macaca mulatta) and bonnet (Macaca radiata) monkeys. Neuropsychologia 49 (7), 2004-2012. http://dx.doi.org 10.1016/j.neuropsychologia.2011.03.030.

Masud, Y., Ajmal, M.A., 2012. Left-handed people in a right-handed world: a phenomenological study. Pak. J. Soc. Clin. Psychol. 10 (1), 49-60.

Mazoyer, B., Zago, L., Jobard, G., Crivello, F., Joliot, M., Perchey, G., et al., 2014. Gaussian mixture modeling of hemispheric lateralization for language in a large sample of healthy individuals balanced for handedness. PLoS One 9 (6), e101165. http://dx.doi.org/10.1371/journal.pone.0101165.

McKenna, A., Hanna, M., Banks, E., Sivachenko, A., Cibulskis, K., Kernytsky, A., et al., 2010. The genome analysis toolkit: a MapReduce framework for analyzing nextgeneration DNA sequencing data. Genome Res. 20 (9), 1297-1303. http://dx.doi. org/10.1101/gr.107524.110.

McManus, I.C., 1985. Handedness, language dominance and aphasia: a genetic model. Psychol. Med. Monogr. Suppl. 8, 1-40.

McManus, I.C., Davison, A., Armour, J.A., 2013. Multilocus genetic models of handedness closely resemble single-locus models in explaining family data and are compatible with genome-wide association studies. Ann. N. Y. Acad. Sci. 1288, 48-58. http://dx.doi.org/10.1111/nyas.12102.

McManus, I.C., Martin, N., Stubbings, G.F., Chung, E.M., Mitchison, H.M., 2004. Handedness and situs inversus in primary ciliary dyskinesia. Proc. Biol. Sci. 271 (1557), 2579-2582. http://dx.doi.org/10.1098/rspb.2004.2881.

Medland, S.E., Duffy, D.L., Wright, M.J., Geffen, G.M., Hay, D.A., Levy, F., et al., 2009a. Genetic influences on handedness: data from 25,732 Australian and Dutch twin families. Neuropsychologia 47 (2), 330-337. http://dx.doi.org/10.1016/j. neuropsychologia.2008.09.005.

Medland, S.E., Lindgren, M., Magi, R., Neale, B.M., Albrecht, E., Esko, T., et al., 2009b. Meta-analysis of GWAS for handedness: results from the ENGAGE consortium. In: Proceedings of Abstract Presented at the 59th Annual Meeting of The American Society of Human Genetics.

Medlar, A., Glowacka, D., Stanescu, H., Bryson, K., Kleta, R., 2013. SwiftLink: paralle MCMC linkage analysis using multicore CPU and GPU. Bioinformatics 29 (4) 413-419. http://dx.doi.org/10.1093/bioinformatics/bts704.

Meguerditchian, A., Vauclair, J., Hopkins, W.D., 2013. On the origins of human handedness and language: a comparative review of hand preferences for bimanual coordinated actions and gestural communication in nonhuman primates. Dev. Psychobiol. 55 (6), 637-650. http://dx.doi.org/10.1002/dev.21150.

NHLBI Exome Sequencing Project (ESP) Exome Variant Server, 2015. Retrieved 13.07.15, from $\langle$ http://evs.gs.washington.edu/EVS/

O'Connell, J.R., Weeks, D.E., 1998. PedCheck: a program for identification of genotype incompatibilities in linkage analysis. Am. J. Hum. Genet. 63 (1), 259-266. http://dx.doi.org/10.1086/301904.

Ocklenburg, S., Beste, C., Arning, L., 2014a. Handedness genetics: considering the phenotype. Front. Psychol. 5, 1300. http://dx.doi.org/10.3389/fpsyg.2014.01300.

Ocklenburg, S., Beste, C., Arning, L., Peterburs, J., Gunturkun, O., 2014b. The ontogenesis of language lateralization and its relation to handedness. Neurosci. Biobehav. Rev. 43, 191-198. http://dx.doi.org/10.1016/j.neubiorev.2014.04.008.

Oldfield, R.C., 1971. The assessment and analysis of handedness: the Edinburgh inventory. Neuropsychologia 9 (1), 97-113.

Perelle, I.B., Ehrman, L., 1994. An international study of human handedness: the data. Behav. Genet. 24 (3), 217-227.

Picard, 2015. Retrieved 25.08.14, from 〈http://broadinstitute.github.io/picard〉.

Preslar, J., Kushner, H.I., Marino, L., Pearce, B., 2014. Autism, lateralisation, and handedness: a review of the literature and meta-analysis. Laterality 19 (1), 64-95. http://dx.doi.org/10.1080/1357650X.2013.772621.

Purcell, S., Neale, B., Todd-Brown, K., Thomas, L., Ferreira, M.A., Bender, D., et al. 2007. PLINK: a tool set for whole-genome association and population-based linkage analyses. Am. J. Hum. Genet. 81 (3), 559-575.

Renteria, M.E., 2012. Cerebral asymmetry: a quantitative, multifactorial, and plastic brain phenotype. Twin Res. Hum. Genet. 15 (3), 401-413. http://dx.doi.org/ 10.1017/thg.2012.13.

Scerri, T.S., Brandler, W.M., Paracchini, S., Morris, A.P., Ring, S.M., Richardson, A.J., et al., 2011. PCSK6 is associated with handedness in individuals with dyslexia. Hum. Mol. Genet. 20 (3), 608-614. http://dx.doi.org/10.1093/hmg/ddq475.

Sherry, S.T., Ward, M.H., Kholodov, M., Baker, J., Phan, L., Smigielski, E.M., Sirotkin, K., 2001. dbSNP: the NCBI database of genetic variation. Nucleic Acids Res. 29 (1), 308-311.

Somers, M., Ophoff, R.A., Aukes, M.F., Cantor, R.M., Boks, M.P., Dauwan, M., et al., 
2015. Linkage analysis in a Dutch population isolate shows no major gene for left-handedness or atypical language lateralization. J. Neurosci. 35 (23), 8730-8736. http://dx.doi.org/10.1523/JNEUROSCI.3287-14.2015.

SureSelect Performance, 2015. Retrieved 15.07.15, from 〈http://www.genomics.agi lent.com/article.jsp?pageId $=3051\rangle$.

Tanaka, S., Kanzaki, R., Yoshibayashi, M., Kamiya, T., Sugishita, M., 1999. Dichotic listening in patients with situs inversus: brain asymmetry and situs asymmetry. Neuropsychologia 37 (7), 869-874.

Tomasi, D., Volkow, N.D., 2012. Resting functional connectivity of language networks: characterization and reproducibility. Mol. Psychiatry 17 (8), 841-854. http://dx.doi.org/10.1038/mp.2011.177.

UCSC Genome Browser on Human, February 2009 (GRCh37/hg19) Assembly, 2015 Retrieved 13.07.15, from $\langle$ http://genome.ucsc.edu/ $\rangle$.

Van Agtmael, T., Forrest, S.M., Del-Favero, J., Van Broeckhoven, C., Williamson, R., 2003. Parametric and nonparametric genome scan analyses for human handedness. Eur. J. Hum. Genet. 11 (10), 779-783. http://dx.doi.org/10.1038/sj. ejhg.5201048.

Van der Auwera, G.A., Carneiro, M.O., Hartl, C., Poplin, R., Del Angel, G., LevyMoonshine, A., et al., 2013. From FastQ data to high-confidence variant calls: the Genome Analysis Toolkit best practices pipeline. Curr. Protoc. Bioinform. 11 (1110). http://dx.doi.org/10.1002/0471250953.bi1110s43 1110 11-11 1033.

Wang, K., Li, M., Hakonarson, H., 2010. ANNOVAR: functional annotation of genetic variants from high-throughput sequencing data. Nucleic Acids Res. 38 (16), e164. http://dx.doi.org/10.1093/nar/gkq603.

Warren, D.M., Stern, M., Duggirala, R., Dyer, T.D., Almasy, L., 2006. Heritability and linkage analysis of hand, foot, and eye preference in Mexican Americans. Laterality 11 (6), 508-524. http://dx.doi.org/10.1080/13576500600761056.

Waters, N.S., Denenberg, V.H., 1994. Analysis of two measures of paw preference in a large population of inbred mice. Behav. Brain Res. 63 (2), 195-204.

Willems, R.M., Van der Haegen, L., Fisher, S.E., Francks, C., 2014. On the other hand: including left-handers in cognitive neuroscience and neurogenetics. Nat. Rev. Neurosci. 15 (3), 193-201. http://dx.doi.org/10.1038/nrn3679. 